\title{
Strain/stress measurement via micro-Raman spectroscopy using predicted deformation potential constants with first-principles calculation
}

\author{
Keita YASHIKI ${ }^{1 *}$ and Hirohisa KIMACHI ${ }^{2 *}$ \\ ${ }^{1}$ Department of Mechanical Engineering, Graduate school of Meijo University, \\ 1-501 Shiogamaguchi, Tempaku-ku, Nagoya, Aichi 468-8502, Japan \\ ${ }^{2}$ Department of Mechanical Engineering, Meijo University, \\ 1-501 Shiogamaguchi, Tempaku-ku, Nagoya, Aichi 468-8502, Japan \\ *Corresponding author’s email: 183430023@ccalumni.meijo-u.ac.jp
}

Introduction: Micro-Raman spectroscopy can quantitatively measure strain/stress in electronic materials used for developing power devices and semiconductors with a submicron spatial resolution. It is necessary to obtain the deformation potential constants to determine the strain/stress components using this technique. However, the experimental determination of these parameters is very difficult because several single-crystal specimens of materials need to be prepared with dimensions that are capable of performing several loading tests. Therefore, the prediction of these parameters using first-principles calculations is useful method to estimate the deformation potential constants.

In this study, we aim to predict the deformation potential constants of single-crystal silicon (Si). Then, we evaluate the stress component distributions in singular stress field in Si under 4-point circular plate bending.

Method: Raman shifts of a single-crystal silicon (Si) were simulated under mechanical loading using firstprinciples calculations with the QUANTUM ESPRESSO open access package. Si belongs to the $O_{\mathrm{h}}$ space group; its Raman spectra correspond to the triply degenerate vibration mode $F_{2 \mathrm{~g}}$. The deformation potential constants $p, q$, and $r$ were calculated based on the relation ${ }^{(1)}$ between the strain components $\varepsilon_{\mathrm{ij}}$ and the Raman shift difference from unstrained Si.

The variation of Raman shifts under 4-point circular plate bending in single-crystal (111) Si using the predicted deformation potential constants were compared with the experimental results.

Result and discussion: Table 1 presents the calculated Raman shifts of unstrained $\mathrm{Si}$, which are triply degenerate, and the deformation potential constants. The calculated parameters were in good agreement with the experimental values. ${ }^{(2)}$

Figure 1 presents the Raman shift variation as a function of strain applied in the $x$-direction for (111) Si under 4-point circular plate bending. The marks represent the Raman shift changes measured by microRaman spectroscopy for each strain value. The lines correspond to the prediction from first-principles calculations. As shown in Fig. 1, the triple degeneracy of the $F_{2 g}$ vibration mode was split when strain was applied. Additionally, the prediction of the slopes of Raman shift change vs the applied strain curves agrees with the experimental values. This demonstrates that the first-principles calculations conducted in this study are useful to predict the deformation potential constants and Raman shifts of strained Si.

We also present the stress component distributions in a singular field around an indentation-induced crack in single-crystal (111) Si. These were calculated using the predicted deformation potential constants and the experimentally obtained Raman shift variation using micro-Raman spectroscopy.

References : (1) S. Venugopalan and A. K. Ramdas, Effect of uniaxial stress on the raman spectra of cubic crystal: $\mathrm{CaF}_{2}, \mathrm{BaF}_{2}$, and $\mathrm{Bil}_{2} \mathrm{GeO}_{20}$, Physical Review B, Vol. 8, No. 2, pp. 717-734 (1973)

(2) E. Anastassakis, A. Cantarero and M. Cardona, Piezo-Raman measurements and anharmonic parameters in silicon and diamond, Physical Review B, Vol. 41, No. 11, pp. 7529-7535 (1990)

Table 1 Predicted Raman shift and deformation potential constants $p, q$, and $r$ of single-crystal silicon.

\begin{tabular}{l|c|c|c}
\hline & Present & Expt. & Error(\%) \\
\hline \hline Raman shift $\left(\mathrm{cm}^{-1}\right)$ & 508.7 & 512.0 & 0.64 \\
\hline$p\left(1 / \mathrm{cm}^{-1}\right)$ & -1.67 & $-1.85^{(2)}$ & 9.72 \\
\hline$q\left(1 / \mathrm{cm}^{-1}\right)$ & -2.08 & $-2.31^{(2)}$ & 9.96 \\
\hline$r\left(1 / \mathrm{cm}^{-1}\right)$ & -0.65 & $-0.71^{(2)}$ & 8.45 \\
\hline
\end{tabular}

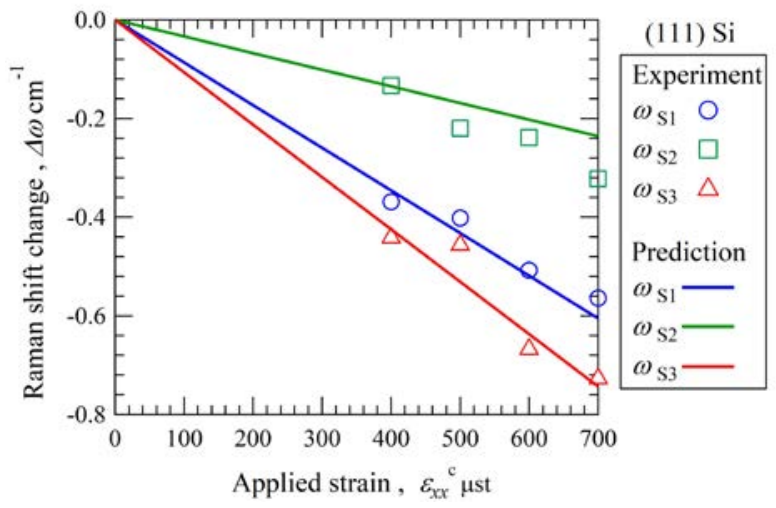

Fig. 1 Raman shift variation of (111) Si under 4-point circular plate bending obtained using the deformation potential constants predicted by first-principles calculations. 